\title{
Common fixed point theorems for strict occasionally weakly compatible mappings in compact metric spaces
}

\author{
Valeriu Popa
}




\title{
COMMON FIXED POINT THEOREMS FOR STRICT OCCASIONALLY WEAKLY COMPATIBLE MAPPINGS IN COMPACT METRIC SPACES
}

\author{
VALERIU POPA \\ Received 18 September, 2012
}

\begin{abstract}
We prove a common fixed point theorem for four two pairs of hybrid mappings in compact metric space satisfying an implicit relations using the concept of strict occasionally weak compatibility which generalize theorems of $[1,4,7,28]$. As an application we obtain a general fixed point theorem for hybrid pairs satisfying a contractive condition of integral type, which is a new result in compact metric spaces.
\end{abstract}

2010 Mathematics Subject Classification: 54H25; 47H10

Keywords: fixed point, pair hybrid, strict occasionally weak compatible mappings, compact space

\section{INTRODUCTION}

Let $(X, d)$ be a metric space. Denote by $B(X)$ the set of all nonempty sets of $X$. As in $[10,11]$ we define the functions $D(A, B)$ and $\delta(A, B)$ by:

$$
\begin{aligned}
D(A, B) & =\inf \{d(a, b): a \in A, b \in B\}, \\
\delta(A, B) & =\sup \{d(a, b): a \in A, b \in B\} .
\end{aligned}
$$

for $A, B \in B(X)$.

If $A$ consists of a single point " $a$ " we write $\delta(A, B)=\delta(a, B)$.

If $B$ consists also of a single point " $b$ " we write $\delta(A, B)=d(a, b)$.

It follows immediately from the definition of $\delta$ that

$$
\begin{gathered}
\delta(A, B)=\delta(B, A) \geq 0, \forall A, B \in B(X), \\
\delta(A, B)=0 \text { implies } A=B=\{a\} .
\end{gathered}
$$

Definition 1 ([10,11]). A sequence $\left\{A_{n}\right\}$ of nonempty sets of $(X, d)$ is said to be convergent to a set $A$ of $X$ if

(i) each point $a \in A$ is the limit of a convergent sequence $\left\{a_{n}\right\}$, where $a_{n} \in A_{n}$ for all $n \in \mathbb{N}$, 
(ii) for any arbitrary $\varepsilon>0$, there exists an integer $m>0$ such that $A_{n} \subset A_{\varepsilon}$ for $n>m$, where $A_{\varepsilon}$ denote the set of all points $x \in X$ for which there exists a point $a \in X$, depending on $x$, such that $d(x, a)<\varepsilon$.

$A$ is said to be the limit of the sequence $\left\{A_{n}\right\}$.

Lemma 1 ([10]). If $\left\{A_{n}\right\}$ and $\left\{B_{n}\right\}$ are sequences in $B(X)$ convergent to $A$ and $B$, respectively, then $\delta\left(A_{n}, B_{n}\right) \rightarrow \delta(A, B)$.

Lemma 2 ([10]). Let $\left\{A_{n}\right\}$ be a sequence in $B(X)$ and $y \in X$ such that $\delta\left(A_{n}, y\right) \rightarrow$ 0 . Then the sequence $\left\{A_{n}\right\}$ converges to the set $\{y\}$ in $B(X)$.

Definition 2. A set valued mapping $F: X \rightarrow B(X)$ is said to be continuous at $x \in$ $X$ if the sequence $\left\{F x_{n}\right\} \in B(X)$ converges to $\{F x\}$, whenever $\left\{x_{n}\right\}$ is a sequence in $X$ converging to $x$ in $X$.

$F$ is said to be continuous at $X$ if it is continuous at every point in $X$.

Let $A$ and $S$ be self mappings of a metric space $(X, d)$. Jungck [12] defined $A$ and $S$ to be compatible if $\lim _{n \rightarrow \infty} d\left(A S x_{n}, S A x_{n}\right)=0$ whenever $\left\{x_{n}\right\}$ is a sequence in $X$ such that $\lim _{n \rightarrow \infty} A x_{n}=\lim _{n \rightarrow \infty} S x_{n}=t$ for some $t \in X$.

A point $x \in X$ is a coincidence point of $A$ and $S$ if $A x=S x$. We denote by $C(A, S)$ the set of all coincidence points of $A$ and $S$.

In [23], Pant defined $A$ and $S$ to be pointwise $R$ - weakly commuting if for all $x \in X$, there exists $R>0$ such that $d(S A x, A S x) \leq R d(A x, S x)$. It has been proved in [24] that pointwise $R$ - weakly commuting is equivalent to commutativity at coincidence points.

Definition 3 ([17]). $A$ and $S$ is said to be weakly compatible if $S A u=A S u$ for $u \in C(A, S)$.

Definition 4 ([2]). $A$ and $S$ is said to be occasionally weakly compatible mappings (briefly owc) if $A S u=S A u$ for some $u \in C(A, S)$.

Remark 1. If $A$ and $S$ are weakly compatible and $C(A, S) \neq 0$ then $A$ and $S$ are owc, but the converse is not true (Example, [2]).

Some fixed point theorems for occasionally weakly compatible mappings are proved in $[2,6-8,16,22,30-32]$ and in other papers.

Definition 5. Let $f:(X, d) \rightarrow(X, d)$ and $F:(X, d) \rightarrow B(X)$ be. Then:

1) a point $x \in X$ is said to be a coincidence point of $f$ and $F$ if $f x \in F x$. We denote by $C(f, F)$ the set of all coincidence points of $f$ and $F$.

2) a point $x \in X$ is said to be a strict coincidence point of $f$ and $F$ if $\{f x\}=$ $F x$. We denote by $S C(f, F)$ the set of all strict coincidence points of $f$ and $F$.

3) a point $x \in X$ is said to be a fixed point of $F$ if $x \in F x$.

4) a point $x \in X$ is said to be a strict fixed point of $F$ if $\{x\}=F x$. 
Definition 6 ([14]). The mappings $f: X \rightarrow X$ and $F: X \rightarrow B(X)$ is said to be $\delta$ - compatible if $\lim _{n \rightarrow \infty} \delta\left(F f x_{n}, f F x_{n}\right)=0$ whenever $\left\{x_{n}\right\}$ is a sequence in $X$ such that $F x_{n} \in B(X), f x_{n} \rightarrow t, F x_{n} \rightarrow\{t\}$ for some $t \in X$.

Definition 7 ([15]). The hybrid pair $f: X \rightarrow X$ and $F: X \rightarrow B(X)$ is weakly compatible if for each $x \in S C(f, F), F f x=f F x$.

Remark 2. If the pair $(f, F)$ is $\delta$ - compatible, then it is weakly compatible but the converse is not true [15].

Definition 8. The hybrid pair $f: X \rightarrow X$ and $F: X \rightarrow B(X)$ is strict occasionally weakly compatible (briefly sowc) if there exists $x \in S C(f, F)$ such that $F f x=f F x$.

Remark 3. If $C(f, F) \neq \phi$ and the pair $(f, F)$ is weakly compatible then the pair $(f, F)$ is owc.

There exists sowc pairs which are not weakly compatible.

Example 1 ([6]). Let $X=[0,2]$ with usual metric. Define $f: X \rightarrow X$ and $F$ : $X \rightarrow B(X)$ by

$$
f x=\left\{\begin{array}{r}
x, x=0 \\
2-x, x \neq 0
\end{array}\right.
$$

and

$$
F x=\left\{\begin{array}{r}
{[0, x], x \leq 1} \\
{[0,2 x], x>1}
\end{array}\right.
$$

Clearly, $C(f, F)=\{0,1\}, S C(f, F)=\{0\}, F f 0=f F 0=\{0\}$ and $F f x \neq f F x$ for all $x \in(0,2]$. Hence, the pair $(f, F)$ is sowc, but it is not weakly compatible.

Remark 4. It is obviously $\{f 0\}=F 0=\{0\}$ and $F 1=[0,1]$. Therefore 0 and 1 are fixed points for $f$ and $F$ and only 0 is a strict point of $f$ and $F$.

\section{PRELIMINARIES}

In [9], Branciari established the following result

Theorem 1. Let $(X, d)$ be a complete metric space, $c \in(0,1)$ and $f: X \rightarrow X$ be a mapping such that for all $x, y \in X$

$$
\int_{0}^{d(f x, f y)} h(t) d t \leq c \int_{0}^{d(x, y)} h(t) d t
$$

where $h:[0, \infty) \rightarrow[0, \infty)$ is a Lebesgue measurable mapping which is summable (i.e. with a finite integral) on each compact subset of $[0, \infty)$ such that for $\varepsilon>0$, $\int_{0}^{\varepsilon} h(t) d t>0$. Then $f$ has a unique fixed point $z \in X$ such that for each $x \in X$, $\lim _{n \rightarrow \infty} f^{n} x=z$.

Recently, Kumar et al. [20] extended Theorem 1 for two compatible mappings. 
Theorem 2. Let $f, g:(X, d) \rightarrow(X, d)$ compatible mappings satisfying the following conditions:

1) $g$ is continuous,

2) $f(X) \subset g(X)$ and

$$
\int_{0}^{d(f x, f y)} h(t) d t \leq c \int_{0}^{d(g x, g y)} h(t) d t,
$$

for all $x, y \in X, c \in(0,1)$, where $h$ is as in Theorem 1.

Then $f$ and $g$ have a unique common fixed point.

Definition 9. Let $X$ be a nonempty set. A symmetric on $X$ is a nonnegative real valued function $D$ on $X \times X$ such that

(i) $D(x, y)=0$ if and only if $x=y$,

(ii) $D(x, y)=D(y, x)$ for any $x, y \in X$.

Some fixed point theorems in metric and symmetric spaces for compatible, weakly compatible and occasionally weakly compatible mappings satisfying a contractive condition of integral type have been established in [3,12, 19,21,29,35] and in other papers.

Let $(X, d)$ be a metric space and $D(x, y)=\int_{0}^{d(x, y)} h(t) d t$, where $h(t)$ is as in Theorem 1. It is proved in [21] and [29] that $D(x, y)$ is a symmetric on $X$. It has also been proved in [21] and [29] that the study of fixed points for mappings satisfying a contractive condition of integral type is reduced to the study of fixed points in symmetric spaces.

The method is not applicable for hybrid pairs.

Definition 10. An altering distance is a mapping $\psi:[0, \infty) \rightarrow[0, \infty)$ which satisfies:

$\left(\psi_{1}\right): \psi$ is increasing and continuous,

$\left(\psi_{2}\right): \psi(t)=0$ if and only if $t=0$.

In [18] a fixed point result involving altering distances have been obtained. Fixed point problem involving altering distances have been studied in $[28,37,38]$ and in other papers.

Definition 11. A weakly altering distance is a mapping $\psi:[0, \infty) \rightarrow[0, \infty)$ which satisfies:

$\left(\psi_{1}\right): \psi$ is increasing,

$\left(\psi_{2}\right): \psi(t)=0$ if and only if $t=0$.

Lemma 3. The function $\psi(t)=\int_{0}^{t} h(x) d x$, where $h(x)$ is as in Theorem 1 is a weakly altering distance.

Proof. The proof follows from Lemma 2.5 [30]. 
Several classical fixed point theorems and common fixed point theorems have recently unified by considering a general condition expressed by an implicit relation $[25,26]$ and other papers.

Actually, the method is used in the study of fixed points in metric spaces, symmetric spaces, quasi - metric spaces, convex metric spaces, reflexive metric spaces, compact metric spaces, paracompact metric spaces, in two and three metric spaces, for single valued functions, hybrid pairs of functions, set - valued functions.

Quite recently, the method is used in the study of fixed points for mappings satisfying a contractive condition of integral type, in fuzzy metric spaces and intuitionistic metric spaces.

In [30] a general fixed point theorem for compatible mappings satisfying an implicit relation has been proved.

In [13] the results from [30] have been improved relaxing compatibility to weak compatibility.

In [27] a general fixed point theorem for weakly compatible mappings in compact metric spaces satisfying an implicit relation is proved.

In [28] a common fixed point theorem for four weakly compatible mappings in compact metric spaces involving an altering distance was proved, which extends the main results of [4] and [37].

Theorem 3 ([28]). Let $f, g, S$ and $T$ be self mappings of a compact metric space $(X, d)$ such that

a) $f(X) \subset T(X)$ and $g(X) \subset S(X)$,

b) the pairs $(f, T)$ is compatible and the pair $(g, S)$ is weakly compatible,

c) $f$ and $S$ are continuous,

d) $\psi(d(f x, g y)) \leq a \psi(d(S x, T y))+b[\psi(d(f x, S x)+\psi(d(g y, T y)]++c[\psi(d(S x, g y)$. $\psi(d(f x, T y)]^{1 / 2}$ for all $x, y \in X, a, b, c \geq 0, a+2 b<1, a+c<1$, and $\psi$ is an altering distance.

Then $f, g, S$ and $T$ have a unique common fixed point in $X$.

Recently, in [5] the authors have proved a new fixed point theorem for mappings satisfying a new type of implicit relation.

The results from [5] are extended in [30] for owc mappings involving altering distances.

In [1] the following theorem is proved.

Theorem 4. Let $I, J$ be two single valued functions from a compact metric space $(X, d)$ into itself and $F, G: X \rightarrow B(X)$ two set-valued functions with $\cup G(X) \subset I(X)$ and $\cup F(X) \subset J(X)$ such that

$$
\begin{aligned}
\psi(\delta(F x, G y))< & \max \{\psi(d(I x, J y)), \psi(\delta(I x, F x)), \psi(\delta(J y, G y)\}, \\
& \min \{\psi(D(I x, G y)), \psi(D(J y, F x))\} \\
& -\omega(\max \{\psi(d(I x, J y)), \psi(\delta(I x, F x)), \psi(\delta(J y, G y)\},
\end{aligned}
$$




$$
\min \{\psi(D(I x, G y)), \psi(D(J y, F x))\})
$$

for all $x, y \in X$, where the right hand side of inequality is positive, $\psi$ is an altering distance and $\omega:[0, \infty) \rightarrow[0, \infty)$ is a continuous function satisfying $0<\omega(r)<r$ for $r>0$.

If the pairs $(I, F)$ and $(J, G)$ are weakly compatible and the functions $F, I$ are continuous, then there exists a unique point $p \in X$ such that $\{p\}=\{I p\}=F p=$ $\{J p\}=G p$.

Remark 5. In the proof of this theorem is used the fact that the function $r-\omega(r)$ is a non-decreasing function.

Some fixed point theorems for hybrid pair in compact metric spaces are proved in $[33,34,36]$ and in other papers.

The purpose of this paper is to extend Theorem 3, Theorem 4 and Theorem 2 [4] for strictly owc mappings satisfying implicit relations and to transfer the study of fixed points for hybrid pairs of mappings satisfying a contractive condition of integral type in compact metric spaces to the study of fixed points in compact metric spaces by altering distances.

\section{IMPLICIT RELATIONS}

Let $\mathscr{F}_{c}$ be the family of all real functions $F: \mathbb{R}_{+}^{6} \rightarrow \mathbb{R}$ satisfying the following conditions:

$\left(\phi_{1}\right) F$ is increasing in variable $t_{1}$ and nonincreasing in variables $t_{2}$ and $t_{4}$,

$\left(\phi_{2}\right)$ If $u \geq 0, v>0, w \geq 0$ such that

$\left(\phi_{2 a}\right) F(u, v, v, u, w, 0) \leq 0$ or

$\left(\phi_{2 b}\right) \quad F(u, v, u, v, 0, w) \leq 0$, then $u<v$ and $u=0$ if $v=0$.

$\left(\phi_{3}\right) \quad F(t, t, 0,0, t, t)>0, \forall t>0$.

Example 2. $F\left(t_{1}, \ldots, t_{6}\right)=t_{1}-a t_{2}-b\left(t_{3}+t_{4}\right)-c\left(t_{5} t_{6}\right)^{1 / 2}$, where $a>0, b, c \geq 0$, $a+2 b<1$ and $a+c<1$.

$\left(\phi_{1}\right)$ : Obviously.

$\left(\phi_{2}\right)$ : Let $u, v>0, w \geq 0$ and $F(u, v, v, u, w, 0)=u-a v-b(u+v) \leq 0$. Then $u \leq \frac{a+b}{1-b} v<v$. Similarly, $F(u, v, u, v, 0, w) \leq 0$ implies $u<v$. If $u=0$, $v>0, w>0$, then $u<v$.

$\left(\phi_{3}\right): F(t, t, 0,0, t, t)=t(1-(a+c))>0, \forall t>0$.

Example 3. $F\left(t_{1}, \ldots, t_{6}\right)=t_{1}^{2}-a t_{2}^{2}-b \frac{t_{3}^{2}+t_{4}^{2}}{1+\min \left\{t_{5}, t_{6}\right\}}$, where $a>0$ and $a+2 b<1$.

$\left(\phi_{1}\right)$ : Obviously.

$\left(\phi_{2}\right)$ : Let $u>0, v>0, w>0$ and $F(u, v, v, u, w, 0)=u^{2}-a v^{2}-b\left(u^{2}+v^{2}\right) \leq 0$ which implies $u^{2} \leq \frac{a+b}{1-b} v^{2}$, hence $u<v$. Similarly, $F(u, v, u, v, 0, w) \leq 0$ implies $u<v$. If $u=0, v>0$ then $u<v$. If $v=0$ then $u=0$. 
$\left(\phi_{3}\right): F(t, t, 0,0, t, t)=t^{2}(1-a)>0, \forall t>0$.

Example 4. $F\left(t_{1}, \ldots, t_{6}\right)=t_{1}^{2}-a t_{2}^{2}-b \frac{t_{3} t_{4}}{1+t_{5} t_{6}}$, where $a>0$ and $a+b<1$.

$\left(\phi_{1}\right)$ : Obviously.

$\left(\phi_{2}\right)$ : Let $u \geq 0, v>0, w \geq 0$ and $F(u, v, v, u, w, 0)=u^{2}-a v^{2}-b u v \leq 0$. Then $f(t)=t^{2}-b t-a \leq 0 \leq$ where $t=\frac{u}{v}$. Since $f(0)=-a<0$ and $f(1)=$ $1-(a+b)>0$, there exists $h \in(0,1)$ such that $f(t)<0$ for $t<h$. Hence $u<v$. Similarly, $F(u, v, u, v, 0, w) \leq 0$ implies $u<v$. If $v=0$ then $u=0$.

$\left(\phi_{3}\right): F(t, t, 0,0, t, t)=t^{2}(1-a)>0, \forall t>0$.

Let $\omega:[0, \infty) \rightarrow[0, \infty)$ with $0<\omega(r)<r$ for $r>0, \omega(0)=0$ and $r-\omega(r)$ is non decreasing.

Example 5.

$F\left(t_{1}, \ldots, t_{6}\right)=t_{1}-\max \left\{t_{2}, t_{3}, t_{4}, \min \left\{t_{5}, t_{6}\right\}\right\}+\omega\left(\max \left\{t_{2}, t_{3}, t_{4}, \min \left\{t_{5}, t_{6}\right\}\right\}\right)$.

$\left(\phi_{1}\right)$ : It follows from the fact that $t-\omega(t)>0$ is a non decreasing function.

$\left(\phi_{2}\right)$ : Let $u \geq 0, v>0, w \geq 0$ and $F(u, v, v, u, w, 0)=u-\max \{u, v\}+\omega(\max \{u, v\}) \leq$ 0 which implies $u-\max \{u, v\}<0$. If $v=0$ then $u-[u-\omega(u)] \leq 0$ which implies $\omega(u) \leq 0$, a contradiction if $u>0$, hence $u=0$. Similarly, $F(u, v, u, v, 0, w) \leq 0$ implies $u<v$ if $v>0$. If $v=0$ then $u=0$.

$\left(\phi_{3}\right): F(t, t, 0,0, t, t)=t-(t-\omega(t))=\omega(t)>0, \forall t>0$.

4. FIXED POINTS FOR SOWC MAPPINGS IN COMPACT METRIC SPACES

Theorem 5. Let $I:(X, d) \rightarrow(X, d)$ and $F:(X, d) \rightarrow B(X)$ be sowc mappings. If $I$ and $F$ have a unique point of strict coincidence $\{z\}=\{I x\}=F x$, then $z$ is the unique common fixed point of $I$ and $F$ which is a strict fixed point for $F$.

Proof. Since $I$ and $F$ are sowc, there exists a point $x \in X$ such that $\{z\}=\{I x\}=$ $F x$ implies $I F x=F I x$. Then, $\{I z\}=\{I I x\} \in I F x=F I x$. Then $u=I x$ is a point of strict coincidence of $I$ and $F$. By hypothesis $u=z$ and $\{z\}=\{I z\}=F z$. Hence $z$ is a common fixed point for $I$ and $F$. Suppose that $v \neq z$ is another common fixed point of $I$ and $F$, which is a strict fixed point for $F$. Then $\{v\}=\{I v\}=F v$. Hence $v$ is a point of strict coincidence of $I$ and $F$, by hypothesis $v=z$.

Theorem 6. Let $(X, d)$ be a metric space and let $I, J: X \rightarrow X$ and $F, G: X \rightarrow$ $B(X)$ such that

$$
\begin{aligned}
& \phi(\psi(\delta(F x, G y)), \psi(d(I x, J y)), \psi(\delta(I x, F x)), \\
& \psi(\delta(J y, G y)), \psi(D(I x, G y)), \psi(D(J y, F x))) \leq 0
\end{aligned}
$$

holds for all $x, y \in X$, where $\phi$ satisfies condition $\left(\phi_{3}\right)$ and $\psi$ is weakly altering distance. Suppose that there exists $x, y \in X$ such that $\{u\}=\{I x\}=F x$ and $\{v\}=$ $\{J y\}=G y$. Then $u$ is the unique point of strict coincidence of $I$ and $F$ and $v$ is the unique point of strict coincidence of $J$ and $G$. 
Proof. First we prove that $I x=J y$. Suppose that $I x \neq J y$. Then by (4.1) we obtain

$$
\phi(\psi(d(I x, J y)), \psi(d(I x, J y)), 0,0, \psi(d(I x, J y)), \psi(d(I x, J x))) \leq 0,
$$

a contradiction of $\left(\phi_{3}\right)$. Hence $d(I x, J y)=0$ which implies $I x=J y$. Thus $\{u\}=$ $\{I x\}=F x=G y=\{J y\}$. Suppose that $z \in X, z \neq x$ such that $\{w\}=\{I z\}=F z$. Then by (4.1) we obtain

$$
\phi(\psi(d(I z, J y)), \psi(d(I z, J y)), 0,0, \psi(d(I z, J y)), \psi(d(I z, J x))) \leq 0,
$$

a contradiction of $\left(\phi_{3}\right)$. Hence $\{w\}=\{I z\}=F z=\{J y\}=G y=F x=\{I x\}=\{u\}$ and $u$ is the unique point of strict coincidence of $I$ and $F$. Similarly, $v$ is the unique point of strict coincidence of $J$ and $G$.

Theorem 7. Let $(X, d)$ be a compact metric space, $I, J: X \rightarrow X$ and $F, G: X \rightarrow$ $B(X)$ satisfying the inequality (4.1) for all $x, y \in X, \phi \in \mathscr{F}_{c}$ satisfies condition $\left(\phi_{3}\right)$ and $\psi$ is weakly altering distance such that $F x \subset J(X)$ and $G x \subset I(X), \forall x \in X$ and the functions $I$ and $F$ are continuous. Then

3) $F$ and I have a strict coincidence point,

4) $G$ and $J$ have a strict coincidence point.

Moreover, if the pairs $(I, F)$ and $(J, G)$ are strict owc, then $I, J, F$ and $G$ have an unique common fixed point which is a strict fixed point for $F$ and $G$.

Proof. Let $m=\inf \{\delta(I x, F x): x \in X\}$. Because $(X, d)$ is compact and $F$ and $I$ are continuous as in $[1,33,34]$ there exists $x_{0} \in X$ such that $\delta\left(I x_{0}, F x_{0}\right)=m$. We prove that $m=0$. Suppose that $m>0$. Since $F x \subset J X, \forall x \in X$, there exists $J y_{0} \in F x_{0}$ and $d\left(I x_{0}, J y_{0}\right) \leq \delta\left(I x_{0}, F x_{0}\right)=m$.

By (4.1) we have

$$
\begin{aligned}
& \phi\left(\psi\left(\delta\left(F x_{0}, G y_{0}\right)\right), \psi\left(d\left(I x_{0}, J y_{0}\right)\right), \psi\left(\delta\left(I x_{0}, F x_{0}\right)\right),\right. \\
& \left.\psi\left(\delta\left(J y_{0}, G y_{0}\right)\right), \psi\left(D\left(I x_{0}, G y_{0}\right)\right), \psi\left(D\left(J y_{0}, F x_{0}\right)\right)\right) \leq 0 .
\end{aligned}
$$

By $\left(\phi_{1}\right)$ we obtain

$$
\begin{aligned}
& \phi\left(\psi\left(d\left(J y_{0}, G y_{0}\right)\right), \psi(m), \psi(m),\right. \\
& \left.\psi\left(\delta\left(J y_{0}, G y_{0}\right)\right), \psi\left(D\left(I x_{0}, G y_{0}\right)\right), 0\right) \leq 0 .
\end{aligned}
$$

Since $\psi(m)>0$, by $\left(\phi_{2 a}\right)$ we obtain

$$
\psi\left(\delta\left(J y_{0}, G y_{0}\right)\right)<\psi(m) .
$$

Since $G x \subset I X, \forall x \in X$, there exists a point $z_{0} \in X$ such that $I z_{0} \in G y_{0}$ and $d\left(I z_{0}, J y_{0}\right) \leq m$. We obtain $\psi(m) \leq \psi\left(\delta\left(I z_{0}, F z_{0}\right)\right) \leq \psi\left(\delta\left(F z_{0}, G y_{0}\right)\right)$.

Then by (4.1) we have

$$
\begin{aligned}
& \phi\left(\psi\left(\delta\left(F z_{0}, G y_{0}\right)\right), \psi\left(d\left(I z_{0}, J y_{0}\right)\right), \psi\left(\delta\left(I z_{0}, F x_{0}\right)\right),\right. \\
& \left.\psi\left(\delta\left(J y_{0}, G y_{0}\right)\right), \psi\left(D\left(I z_{0}, G y_{0}\right)\right), \psi\left(D\left(J y_{0}, F z_{0}\right)\right)\right) \leq 0 .
\end{aligned}
$$


By $\left(\phi_{1}\right)$ we obtain

$$
\begin{aligned}
& \phi\left(\psi\left(\delta\left(I z_{0}, F z_{0}\right)\right), \psi(m), \psi\left(\delta\left(I z_{0}, F z_{0}\right)\right),\right. \\
& \left.\psi(m), 0, \psi\left(D\left(J y_{0}, F z_{0}\right)\right)\right) \leq 0 .
\end{aligned}
$$

By $\left(\phi_{2 b}\right)$ we have

$$
\psi\left(\delta\left(I z_{0}, F z_{0}\right)\right)<\psi(m) .
$$

Hence, $\psi(m) \leq \psi\left(\delta\left(I z_{0}, F z_{0}\right)\right)<\psi(m)$, a contradiction. Hence $m=0$ and $\psi(m)=0$. By $(4.2) \psi\left(\delta\left(J y_{0}, G y_{0}\right)\right)=0$ which implies $\left\{J y_{0}\right\}=G y_{0}$. Therefore $\left\{I x_{0}\right\}=F x_{0}=\left\{J y_{0}\right\}=G y_{0}=\{p\}$. Hence, $x_{0}$ is a strict coincidence point of $I$ and $F$ and $y_{0}$ is a strict coincidence point of $J$ and $G$.

By Theorem 6,p is the unique point of strict coincidence of $I$ and $F$ and also $p$ is the unique point of strict coincidence of $J$ and $G$.

If $(I, F)$ and $(J, G)$ are sowc, then by Theorem $5, p$ is the unique common fixed point of $I, J, F$ and $G$, which is a strict fixed point for $F$ and $G$.

Remark 6. (1) By Example 2 and Theorem 7 we obtain a generalization of Theorem 3.

(2) By Example 5 and Theorem 7 we obtain a generalization of Theorem 4.

If $\psi(t)=t$ by Theorem 7 we obtain

Theorem 8. Let $(X, d)$ be a compact metric space, $I, J: X \rightarrow X$ and $F, G: X \rightarrow$ $B(X)$ satisfying the following conditions:

a) $F x \subset J(X)$ and $G x \subset I(X), \forall x \in X$,

b) the functions $I$ and $F$ are continuous,

c) $\phi(\delta(F x, G y), d(I x, J y), \delta(I x, F x), \delta(J y, G y), D(I x, G y), D(J y, F x)) \leq$ 0 , for all $x, y \in X$ and $\phi \in \mathcal{F}_{c}$. Then:

d) $F$ and $I$ have a strict coincidence point,

e) $G$ and $J$ have a strict coincidence point.

Moreover, if the pairs $(I, F)$ and $(J, G)$ are strict owc, then $I, J, F$ and $G$ have an unique common fixed point which is a strict fixed point for $F$ and $G$.

Remark 7. If $I, J, F$ and $G$ are self mappings of $(X, d)$ then by Theorem 7 we obtain a generalization of Theorem 4.1 [7].

Corollary 1. Let $(X, d)$ be a compact metric space, $I, J: X \rightarrow X$ and $F, G: X \rightarrow$ $B(X)$ satisfying the following conditions:

a) $F(X) \subset J(X)$ and $G(X) \subset I(X), \forall x \in X$,

b) the functions $I$ and $F$ are continuous,

c) $\phi(\delta(F x, G y)) \leq$ $a d(I x, J y)+b[\delta(I x, F x)+\delta(J y, G y)]+c[D(I x, G y) \cdot D(J y, F x)]^{1 / 2}$, for all $x, y \in X$, where $a>0, b, c \geq 0, a+2 b<1$ and $a+c<1$. Then

d) $F$ and $I$ have a strict coincidence point, 
e) $G$ and $J$ have a strict coincidence point.

Moreover, if the pairs $(I, F)$ and $(J, G)$ are strict owc, then $I, J, F$ and $G$ have an unique common fixed point which is a strict fixed point for $F$ and $G$.

Proof. The proof follows by Theorem 8 and Example 2.

Example 6. Let $X=[0,1]$ endowed with the Euclidean metric $d$. We define

$$
\begin{aligned}
& F x=\left\{\frac{1}{2}\right\}, x \in[0,1] \\
& G x=\left\{\begin{array}{c}
\frac{1}{2}, x \in\left[0, \frac{1}{2}\right] \\
\left(\frac{1}{4}, \frac{1}{2}\right], x \in\left(\frac{1}{2}, 1\right]
\end{array}\right. \\
& I x=\left\{\begin{array}{c}
\frac{2 x+1}{4}, x \in\left[0, \frac{1}{2}\right] \\
\frac{1}{2}, x \in\left(\frac{1}{2}, 1\right]
\end{array} \quad J x=\left\{\begin{array}{c}
1-x, x \in\left[0, \frac{1}{2}\right] \\
0, x \in\left(\frac{1}{2}, 1\right]
\end{array}\right.\right.
\end{aligned}
$$

Then we have

$$
F X=\left\{\frac{1}{2}\right\}, \quad G X=\left[\frac{1}{4}, \frac{1}{2}\right], \quad I X=\left[\frac{1}{4}, \frac{1}{2}\right], \quad J X=\{0\} \cup\left[\frac{1}{2}, 1\right] .
$$

Hence $F(X) \subset J(X), G(X) \subset I(X)$.

$I$ and $F$ are continuous.

$$
\begin{aligned}
J\left(\frac{1}{2}\right) & =G\left(\frac{1}{2}\right)=\left\{\frac{1}{2}\right\}, & I\left(\frac{1}{2}\right) & =F\left(\frac{1}{2}\right)=\left\{\frac{1}{2}\right\}, \\
I F\left(\frac{1}{2}\right) & =I\left(\frac{1}{2}\right)=\frac{1}{2}, & F I\left(\frac{1}{2}\right) & =F\left(\frac{1}{2}\right)=\left\{\frac{1}{2}\right\}, \\
J G\left(\frac{1}{2}\right) & =J\left(\frac{1}{2}\right)=\frac{1}{2}, & G J\left(\frac{1}{2}\right) & =G\left(\frac{1}{2}\right)=\left\{\frac{1}{2}\right\} .
\end{aligned}
$$

Hence, $(I, F)$ and $(J, G)$ are strict owc.

If $x \in[0,1]$ and $y \in\left[0, \frac{1}{2}\right]$ then $\delta(F x, G y)=0$. $\frac{1}{2}$.

If $y \in\left(\frac{1}{2}, 1\right]$, then $\delta(F x, G y)=\delta\left(\left\{\frac{1}{2}\right\},\left(\frac{1}{4}, \frac{1}{2}\right]\right)=\frac{1}{4}$ and $d(I x, J y)=d\left(\left\{\frac{1}{2}\right\}, 0\right)=$

Hence the condition $c$ ) of Corollary 1 is satisfied for $a>\frac{1}{2}, a+2 b<1, a+c<1$.

Hence $I, J, F$ and $G$ have an unique common fixed point $x=\frac{1}{2}$, which is a strict fixed point for $F$ and $G$.

5. AltERING DISTANCE AND FIXED POINTS FOR HYBRID PAIRS SATISFYING A CONTRACTIVE CONDITION OF INTEGRAL TYPE

Theorem 9. Let $(X, d)$ be a compact metric space, $I, J:(X, d) \rightarrow(X, d)$ and $F, G: X \rightarrow B(X)$ satisfying the following conditions:

1) $F x \subset J(X)$ and $G x \subset I(X), \forall x \in X$,

2) the functions $I$ and $F$ are continuous, 
3) $\phi\left(\int_{0}^{\delta(F x, G y)} h(t) d t, \int_{0}^{d(I x, J y)} h(t) d t, \int_{0}^{\delta(I x, F x)} h(t) d t\right.$,

$$
\left.\int_{0}^{\delta(J y, G y)} h(t) d t, \int_{0}^{D(I x, G y)} h(t) d t, \int_{0}^{D(J y, F x)} h(t) d t\right) \leq 0,
$$

for all $x, y \in X$, where $\phi \in \mathscr{F}_{c}$ and $h(t)$ is as in Theorem 1. Then:

4) $F$ and $I$ have a strict coincidence point,

5) $G$ and $J$ have a strict coincidence point.

Moreover, if the pairs $(I, F)$ and $(J, G)$ are strict owc, then $I, J, F$ and $G$ have an unique common fixed point which is a strict fixed point for $F$ and $G$.

Proof. As in Lemma 3 we have

$$
\begin{array}{rlrl}
\int_{0}^{\delta(F x, G y)} h(t) d t & =\psi(\delta(F x, G y)), & & \int_{0}^{d(I x, J y)} h(t) d t=\psi(d(I x, J y)), \\
\int_{0}^{\delta(I x, F x)} h(t) d t=\psi(\delta(I x, F x)), & & \int_{0}^{\delta(J y, G y)} h(t) d t=\psi(\delta(J y, G y)), \\
\int_{0}^{D(I x, G y)} h(t) d t=\psi(D(I x, G y)), & \int_{0}^{D(J y, F x)} h(t) d t=\psi(D(J y, F x)) .
\end{array}
$$

Then by 3) we obtain

$$
\begin{aligned}
& \phi(\psi(\delta(F x, G y)), \psi(d(I x, J y)), \psi(\delta(I x, F x)), \\
& \psi(\delta(J y, G y)), \psi(D(I x, G y)), D(J y, F x)) \leq 0 .
\end{aligned}
$$

By Lemma $3 \psi(t)$ is a weakly altering distance. Hence the conditions of Theorem 7 are satisfied and the conclusion of Theorem 9 follows from Theorem 7.

Remark 8. If $h(t)=1$, by Theorem 9 we obtain Theorem 8 .

By Theorem 9 and Example 2 - 5 we obtain particular results for mappings satisfying implicit relations in compact metric space. For example, by Theorem 9 and Example 2 we obtain

Corollary 2. Let $(X, d)$ be a compact metric space, $I, J:(X, d) \rightarrow(X, d), F, G$ : $X \rightarrow B(X)$ satisfying conditions (1) and (2) of Theorem 9 and

$$
\begin{aligned}
& \int_{0}^{\delta(F x, G y)} h(t) d t \leq a \int_{0}^{d(I x, J y)} h(t) d t+b\left[\int_{0}^{\delta(I x, F x)} h(t) d t+\right. \\
& \left.\int_{0}^{\delta(J y, G y)} h(t) d t\right]+c\left(\int_{0}^{D(I x, G y)} h(t) d t \int_{0}^{D(J y, F x)} h(t) d t\right)^{1 / 2} \leq 0,
\end{aligned}
$$

for all $x, y \in X$, where $h(t)$ is as in Theorem 1. Then:

a) $F$ and $I$ have a strict coincidence point,

b) $G$ and $J$ have a strict coincidence point.

Moreover, if the pairs $(I, F)$ and $(J, G)$ are strict owc, then $I, J, F$ and $G$ have an unique common fixed point which is a strict fixed point for $F$ and $G$. 


\section{REFERENCES}

[1] M. E. Abd EI-Monsef, H. M. Abu-Donia, and K. Abd-Rabou, "Common fixed point theorems under a new contractive type mappings," J. Egypt. Math. Soc., vol. 17, no. 2, pp. 125-133, 2009.

[2] M. A. Al-Thagafi and N. Shahzad, "Generalized $I$-nonexpansive selfmaps and invariant approximations," Acta Math. Sin., Engl. Ser., vol. 24, no. 5, pp. 867-876, 2008.

[3] A. Aliouche, "A common fixed point theorem for weakly compatible mappings in symmetric spaces satisfying a contractive condition of integral type," J. Math. Anal. Appl., vol. 322, no. 2, pp. 796-802, 2006.

[4] A. Aliouche, "A common fixed point theorem for weakly compatible maps in compact metric spaces satisfying an implicit relation," Sarajevo J. Math., vol. 3, no. 1, pp. 1-6, 2007.

[5] A. Aliouche and A. Djoudi, "Common fixed point theorems for mappings satisfying an implicit relation without decreasing assumption," Hacet. J. Math. Stat., vol. 36, no. 1, pp. 11-18, 2007.

[6] A. Aliouche and V. Popa, "General common fixed point theorems for occasionally weakly compatible hybrid mappings and applications," Novi Sad J. Math., vol. 39, no. 1, pp. 89-109, 2009.

[7] A. Aliouche and V. Popa, "Common fixed point theorems for occasionally weakly compatible mappings in compact metric spaces," Acta Univ. Apulensis, Math. Inform., vol. 28, pp. 203-214, 2011.

[8] H. Bouhadjera and C. Godet-Thobie, "Common fixed point theorems for occasionally weakly compatible maps," Acta Math. Vietnam., vol. 36, no. 1, pp. 1-17, 2011.

[9] A. Branciari, "A fixed point theorem for mappings satisfying a general contractive condition of integral type," Int. J. Math. Math. Sci., vol. 29, no. 9, pp. 531-536, 2002.

[10] B. Fisher, "Common fixed point for mappings and set valued mappings," Rostok Math. Kollok., vol. 18, pp. 69-77, 1981.

[11] B. Fisher and S. Sessa, "Two common fixed point theorems for weakly commuting mappings," Period. Math. Hung., vol. 20, no. 3, pp. 207-218, 1989.

[12] F. Gu and H. Ye, "Common fixed point theorems of Altman integral type mappings in $G$-metric spaces," Abstr. Appl. Anal., vol. 2012, p. 13, 2012.

[13] M. Imdad, S. Kumar, and M. S. Khan, "Remarks on some fixed point theorems satisfying implicit relations," Rad. Mat., vol. 11, no. 1, pp. 135-143, 2002.

[14] G. Jungck and B. E. Rhoades, "Some fixed point theorems for compatible maps." Int. J. Math. Math. Sci., vol. 16, no. 3, pp. 417-428, 1993.

[15] G. Jungck and B. E. Rhoades, "Fixed points for set valued functions without continuity," Indian J. Pure Appl. Math., vol. 29, no. 3, pp. 227-238, 1998.

[16] G. Jungck and B. E. Rhoades, "Fixed point theorems for occasionally weakly compatible mappings," Fixed Point Theory, vol. 7, no. 2, pp. 287-296, 2006.

[17] G. Jungck, "Compatible mapping and common fixed points," Int. J. Math. Math. Sci., vol. 9, pp. 771-779, 1986.

[18] M. S. Khan, M. Swaleh, and S. Sessa, "Fixed point theorems by altering distances between the points," Bull. Aust. Math. Soc., vol. 30, pp. 1-9, 1984.

[19] J. K. Kohli and S. Vashistha, "Common fixed point theorems for compatible and weakly compatible mappings satisfying general contractive type conditions," Stud. Cercet. Ştiinţ., Ser. Mat., Univ. Bacău, vol. 16, pp. 33-42, 2006.

[20] S. Kumar, R. Chugh, and R. Kumar, "Fixed point theorems for compatible mappings satisfying a contractive condition of integral type," Soochow J. Math., vol. 33, no. 2, pp. 181-185, 2007.

[21] M. Mocanu and V. Popa, "Some fixed point theorems for mappings satisfying implicit relations in symmetric spaces," Libertas Math., vol. 28, pp. 1-13, 2008.

[22] B. D. Pant and S. Chauhan, "Common fixed point theorem for occasionally weakly compatible mappings in Menger space," Surveys in Mathematics and its Applications, vol. 6, pp. 1-7, 2011. 
[23] R. P. Pant, "Common fixed points of noncommuting mappings," J. Math. Anal. Appl., vol. 188, no. 2, pp. 436-440, 1994.

[24] R. P. Pant, "Common fixed points for four mappings," Bull. Calcutta Math. Soc., vol. 9, pp. 281286, 1998.

[25] V. Popa, "Fixed point theorems for implicit contractive mappings," Stud. Cercet. Ştiinţ., Ser. Mat., Univ. Bacău, vol. 7, pp. 127-133, 1997.

[26] V. Popa, "Some fixed point theorems for compatible mappings satisfying an implicit relation," Demonstr. Math., vol. 32, no. 1, pp. 157-163, 1999.

[27] V. Popa, "A general fixed point theorem for weakly compatible mappings in compact metric spaces," Turk. J. Math., vol. 25, no. 4, pp. 465-474, 2001.

[28] V. Popa, "A fixed point theorem for four compatible mappings in compact metric spaces," U. P. B. Bull. Ser. A, vol. 63, no. 4, pp. 43-46, 2001.

[29] V. Popa and M. Mocanu, "A new viewpoint in the study of fixed points for mappings satisfying a contractive condition of integral type," Bul. Inst. Politeh. Iaşi, Sect. Mat. Mec. Teor. Fiz., vol. 53, no. 57, pp. 269-282, 2007.

[30] V. Popa and M. Mocanu, "Altering distances and common fixed point under implicit relation," Hacettepe J. Math. Statistics, vol. 38, no. 3, pp. 329-337, 2009.

[31] H. Rahimi and G. S. Rad, "Some fixed point results in metric spaces," J. Basic Appl. Sci. Res., vol. 2, no. 9, pp. 9301-9308, 2012.

[32] H. Rahimi, P. Vetro, and G. S. Rad, "Some common fixed point results for weakly compatible mappings in cone metric type space," Miskolc Math. Notes, vol. 14, no. 1, pp. 233-243, 2013.

[33] R. A. Rashwan and M. A. Ahmed, "Common fixed points for $\delta$-compatible mappings," Southwest J. Pure Appl. Math., vol. 1, pp. 51-61, 1996.

[34] R. A. Rashwan and M. A. Ahmed, "Common fixed points for weakly $\delta$-compatible mappings," Italian J. Pure Appl. Math., vol. 8, pp. 33-44, 2000.

[35] B. E. Rhoades, "Two fixed-point theorems for mappings satisfying a general contractive condition of integral type," Int. J. Math. Math. Sci., vol. 2003, no. 63, pp. 4007-4013, 2003.

[36] T. K. Samanta and S. Mohinta, "Common fixed point theorems for hybrid maps in nonArchimedean fuzzy metric spaces," J. Appl. Math. Inform., vol. 31, no. 1-2, pp. 155-164, 2013.

[37] K. P. R. Sastry and G. V. R. Babu, "Fixed point theorems in metric spaces by altering distances," Bull. Calcutta Math. Soc., vol. 90, no. 3, pp. 175-182, 1998.

[38] K. P. R. Sastry and G. V. R. Babu, "Some fixed point theorems by altering distances between the points," Indian J. Pure Appl. Math., vol. 30, no. 6, pp. 641-647, 1999.

Author's address

Valeriu Popa

Vasile Alecsandri University of Bacău, Department of Mathematics, Informatics and Educational

Sciences, 157 Calea Mărăşeşti, 600115 Bacău, Romania

E-mail address: vpopa@ub.ro 